\title{
Development of a C18 Supercritical Fluid Chromatography-Tandem Mass Spectrometry Methodology for the Analysis of Very-Long- Chain Polyunsaturated Fatty Acid Lipid Matrices and Its Application to Fish Oil Substitutes Derived from Genetically Modified Oilseeds in the Aquaculture Sector
}

\author{
Richard Broughton,* Douglas R. Tocher, and Mónica B. Betancor
}

Cite This: ACS Omega 2020, 5, 22289-22298

Read Online

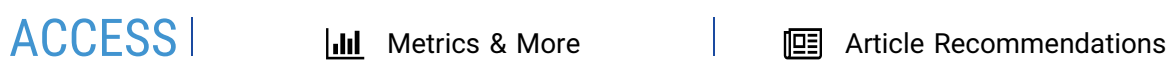

Supporting Information

ABSTRACT: Lipidomics methodologies traditionally utilize either reverse phase- or hydrophilic interaction liquid chromatography-type separations; however, supercritical fluid chromatography can offer a rapid normal phase type separation while reducing the dependence on organic solvents. However, normal phase type lipid separations typically lack pronounced intraclass separation, which is problematic for complex lipidomes containing very-long-chain polyunsaturated fatty acids, especially those from genetically modified organisms. A high-strength silica C18 method was developed, which benefitted from discrete class separation, as well as displaying intraclass selectivity sufficient for profiling flesh of salmon fed with a diet supplemented with oil from the genetically engineered oilseed Camelina sativa, a terrestrial oilseed

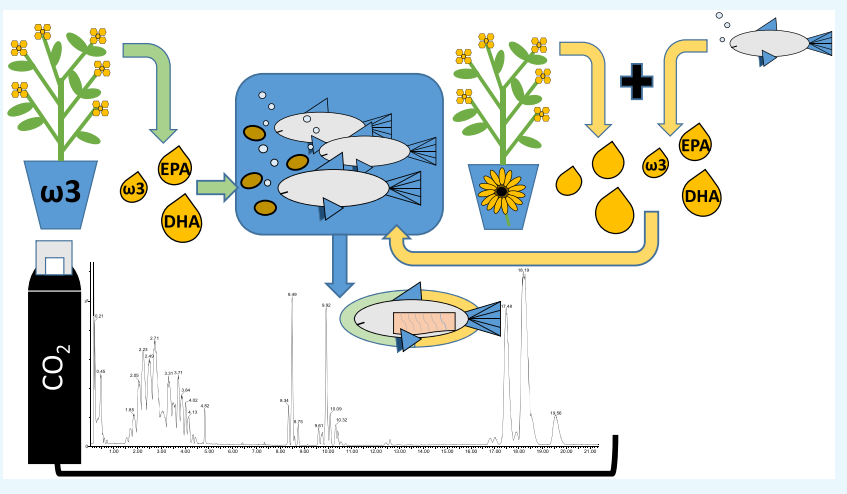
with a fish oil-type profile. Salmon fed a diet containing this Camelina oil were found to have flesh enriched in triacylglycerols and phospholipids containing 18:3, 20:5, and 22:6, whereas salmon fed the control diet were differentiated by shorter chain plant-type fatty acids integrated within complex lipids. Coupled with active scanning quadrupole technology, data acquisition was enhanced, allowing for fragmentation data to be acquired in a data independent fashion, permitting acyl chain identification of resolved isomers. Therefore, we have developed a method, which is amenable for lipidomics studies of complex lipidomes, specifically those altered by synthetic biology approaches.

\section{INTRODUCTION}

The field of lipidomics is one of the most recent omics techniques, evolving from metabolomics, and addressing biological problems, which were not extensively provisioned for by metabolomics, traditionally focused toward the polar end of the metabolite spectrum. Lipidomics has predominantly been carried out using both reverse phase and normal phase hydrophilic interaction liquid chromatography (HILIC), depending on whether separation by the acyl chain or head group was required. Supercritical fluid chromatography (SFC) has been utilized for several decades; however, it is only relatively recently that it has been promoted as a mature analytical technique. SFC is highly amenable to nonpolar compounds, with supercritical $\mathrm{CO}_{2}$ displaying the polarity of hexane, as well as demonstrating a low viscosity allowing for higher flow rates with small particle columns, resulting in more efficient separations when compared with normal phase chromatography, its closest chromatographic relative. With recent advances in hardware, the ability to consistently reproduce chromatographic behavior is now possible with, for example, the inclusion of automatic back pressure regulators $(A B P R)$ and new pumps designed specifically for supercritical fluids.

However, SFC has not seen widespread adoption within the lipidomics field and to date, only a few applications have been described, ranging from the silica-like bridged ethylene hybrid $(\mathrm{BEH})$ column, ${ }^{1,2}$ method development across multiple columns, ${ }^{3,4}$ and $\mathrm{C} 18$ columns. ${ }^{5,6}$ Currently, the BEH or similar column is used for lipid class separation, whereas the highstrength silica (HSS) C18 column has been used predominantly for neutral lipids, such as for free fatty acid and triacylglycerol analysis. ${ }^{7}$ One disadvantage to using a $\mathrm{BEH}$

Received: June 3, 2020

Accepted: July 31, 2020

Published: August 24, 2020 
column for lipid separation is the lack of intraclass separation, whereby all isomers of a class coelute. For quantitation purposes, this can allow for more accurate estimations, but for isomer identification and quantification, this proves to be challenging for complex lipidomes. An alternative to this is to use an HSS C18 column for lipidome profiling, which provides additional selectivity based on the acyl chain. A C18 column has been used for this purpose; however, only a limited number of compounds were studied, with phospholipid peaks unoptimized and broad. ${ }^{6}$ It is our aim to develop a method capable of resolving classes based on the head group and offering selectivity through the acyl function. This offers the benefit of allowing for more robust mass spectral acquisition and the subsequent identification of the fatty acids bound to individual isomers. By separating compounds in time, datadriven acquisitions (DDA) or non-DDA methodologies, such as $\mathrm{MS}^{\mathrm{e}}$, allow for purer spectral acquisition for target identification. As $\mathrm{MS}^{\mathrm{e}}$ identifies product ion spectra from a precursor based on the correlation between the elution times, much like deconvolution on electron ionization GC-MS systems, separation of compounds by time is required to elucidate product ion spectra. An improvement in this technique utilizes a scanning resolving quadrupole, which is capable of isolating and fragmenting coeluting compounds of different molecular masses. This can allow for more precise lipid assignment by identifying the fatty acyl chains bound to the complex lipids in an unbiased fashion. This can yield important information, especially in complex lipidomes containing very-long-chain polyunsaturated fatty acids (VLCPUFAs), where multiple fatty acid isomers may be present.

Methodologies to separate multiple isomers are important within the field of metabolic engineering as alterations to a metabolic pathway may yield unexpected byproducts. It is also essential to validate that the compounds of interest are located within the right lipid pool, and possibly as the right regioisomer as is the case with infant formula. ${ }^{8,9}$ In cases where polyunsaturated fatty acid metabolism is altered, the number of potential lipid isomers increases substantially. This is the case with both transgenic Camelina sativa, ${ }^{10}$ Brassica napus, ${ }^{11}$ and certain algal species, ${ }^{12}$ whereby fatty acid metabolism has been extended to produce the very-longchain polyunsaturated fatty acids, eicosapentaenoic acid (EPA), and docosahexaenoic acid (DHA). To fully understand the altered lipid metabolism of these organisms, a more comprehensive approach is needed. Knowledge of how and where novel lipids are formed will provide insights into the formation of these fatty acids and to the potential location of bottlenecks and substrate specificities of heterologously expressed enzymes. These novel producers of VLCPUFA are seen as an alternative source of these fatty acids for both the human diet directly, and potentially more importantly, indirectly as a feed ingredient, as fish oil substitutes for the aquaculture industry. ${ }^{13}$ These sources intend to make aquaculture more sustainable by easing the burden of VLCPUFA production from the aquaculture industry itself taken from both the byproducts and from the use of smaller fish species to a more sustainable terrestrial source. These terrestrial sources may eventually supplant the current use of non-VLCPUFA-containing oils, such as those from oilseed rape. However, methodologies need to be developed to determine the parity of these feeds and whether comparable outcomes are observed for fish health and for flesh quality, with one measure being the comparison of the lipidomes.

To this extent, we seek to develop a method capable of separating a broad range of lipids, one tailored to the increased complexity of VLCPUFA matrices, which offers the ability to collect increased structural information, such as fatty acid composition. We acknowledge, however, that a single method will never truly capture all the available information, and as such see this methodology as part of a pipeline, as more targeted approaches, informed by the untargeted SFC methodology, address analytical challenges such as sn positional analysis of phospholipids within a specific class.

\section{RESULTS AND DISCUSSION}

C18 SFC/ESI-MS/MS Method Development. Several columns were trialed as a starting point to ascertain whether any column chemistries available for SFC applications were capable of intracompound selectivity. Mobile phases were optimized based on prior works with BEH columns, ${ }^{1,2}$ with the best peak shapes achieved with a modifier comprising methanol/acetonitrile $(80: 20, \mathrm{v} / \mathrm{v})$. It was also found that an increased concentration of ammonium formate at $0.15 \%(\mathrm{w} / \mathrm{v})$ provided an optimal peak shape as did the addition of $0.1 \%$ (v/ v) formic acid, which also assists with peak retention time stability for $\mathrm{BEH}$ columns. ${ }^{2}$ Columns such as the $\mathrm{BEH}$ and torus diol columns separate predominantly based on the compound class with intraclass separation being limited. For lipidomes that contain large quantities of polyunsaturated fatty acids, isomer separation and peak identification proved difficult to achieve even with technologies such as $\mathrm{MS}^{\mathrm{e}}$ and Sonar, which permit the deconvolution of $\mathrm{MS}^{2}$ spectra by both time and precursor mass. It was found that a C18 HSS column was capable of separating by class and offered intraclass specificity based on the acyl chains. The initial method utilized a C18 HSS $150 \times 2.1 \mathrm{~mm}$ column, and this was found to provide suitable separation within neutral lipid fractions and for phospholipids. However, choline-containing lipids, such as phosphatidylcholine (PC), suffer from broader peak shapes but still achieve intraclass separation suitable for isomer separation and peak identification.

Unfortunately, silica-based columns suffer from silyl ether formation, ${ }^{14}$ which is the result of SFC mobile phases excluding water from the system. This results in the gradual methylation of the silanol groups and subsequent changes in retention times, selectivity, and peak shapes. The uncapped silica is, however, required for the effective separation of phospholipids under SFC conditions, which is analogous to normal phase chromatography. This was demonstrated through the use of a highly endcapped Acquity BEH C18 column (Figure S1), which resulted in poor phospholipid selectivity under SFC conditions. Second, the production of silyl ethers under SFC conditions can be reversed by the addition of water to the mobile phase, ${ }^{14}$ which effectively hydrolyses the ethers, yielding free silanol groups. The addition of water to the mobile phase also improved the peak shape and separation within the phospholipid class, most notably for phosphatidylinositol (PI) and PC, where the former is needed to improve the peak shape substantially and the latter to improve intraclass selectivity. However, the column usually exists in a nonequilibrated state, with the silanols partly etherified, the extent of which depends on the mobile phase and storage conditions. With subsequent runs with water in the mobile phase, PC and choline-containing compounds adhere 
more strongly to the vacant silanol groups, extending their retention time with subsequent runs (Figure S2). Remethylation of the column is much slower and cannot be achieved in nonaqueous conditions in a standard LC run time frame. The column was stored and run under nonaqueous conditions for several weeks, and PC was found to elute near its original retention time, albeit suffering from poorer intraclass separation. Therefore, columns need to be kept in either a completely methylated or nonmethylated state to ensure reproducibility with regards to lipids.

To address this issue, a shorter C18 column was used $(50 \times$ $2.1 \mathrm{~mm})$, solely with solvents containing $5 \%$ water. This ensured the column remained silyl ether free and resulted in more stable retention times. The short column resulted in little decrease in intraclass selectivity for phospholipids; however, intraclass selectivity within neutral lipids was reduced when compared with the $15 \mathrm{~cm} \mathrm{C18}$ column (Table 1 and Figure

Table 1. Calculation of Selectivity Factors to Compare Both the Short and Long C18 Columns

\begin{tabular}{|c|c|c|c|c|}
\hline \multirow[b]{2}{*}{ ID } & \multicolumn{2}{|c|}{$\mathrm{C}_{18}(5 \mathrm{~cm})$} & \multicolumn{2}{|c|}{$\mathrm{C}_{18}(15 \mathrm{~cm})$} \\
\hline & time & selectivity & time & selectivity \\
\hline PE 38:6 & 9.59 & 0.00 & 14.11 & 0.00 \\
\hline PE 36:4a & 9.61 & 1.07 & 14.09 & -1.78 \\
\hline PE 36:3 & 9.73 & 9.45 & 14.21 & 9.06 \\
\hline PE 38:5a & 9.73 & 10.59 & 14.22 & 11.32 \\
\hline PE 34:2 & 9.76 & 8.14 & 14.19 & 8.23 \\
\hline PE 38:4 & 9.92 & 19.20 & 14.41 & 25.32 \\
\hline PE 36:2a & 9.94 & 29.41 & 14.39 & 31.33 \\
\hline PE 38:5b & 9.94 & 31.51 & 14.38 & 32.11 \\
\hline PE 34:1 & 9.96 & 23.71 & 14.38 & 22.29 \\
\hline PE 36:4b & 9.96 & 22.94 & 14.37 & 23.52 \\
\hline PE $36: 2 b$ & 10.09 & 33.17 & 14.51 & 35.60 \\
\hline PE 38:5c & 10.09 & 38.59 & 14.51 & 41.46 \\
\hline PE $38: 3$ & 10.13 & 40.84 & 14.58 & 45.02 \\
\hline PE 36:1 & 10.32 & 43.83 & 14.71 & 44.70 \\
\hline TAG 50:6 & 1.7 & 0.00 & 7.32 & 0.00 \\
\hline TAG 50:5 & 1.87 & 5.50 & 7.56 & 9.03 \\
\hline TAG $48: 2$ & 1.85 & 3.86 & 7.45 & 5.43 \\
\hline TAG 50:2 & 2.23 & 12.37 & 7.97 & 25.73 \\
\hline TAG 54:7 & 2.28 & 12.12 & 8.15 & 32.52 \\
\hline TAG 52:2 & 2.74 & 25.71 & 8.46 & 41.64 \\
\hline TAG 56:7 & 2.79 & 27.12 & 8.6 & 53.47 \\
\hline TAG 56:2 & 3.71 & 59.39 & 9.3 & 98.82 \\
\hline TAG 60:7 & 3.71 & 67.27 & 9.38 & 92.98 \\
\hline
\end{tabular}

S3). By comparing the BEH column with the C18 HSS $(5 \mathrm{~cm})$ column, with phosphatidylethanolamine (PE) as the reference class, it can be seen that much greater intraclass separation is achieved (Figure 1) with the C18 column, separating compounds based on the carbon number and number of double bonds more efficiently. The C18 column was also capable of separating fatty acid isomers as demonstrated with $m / z$ 766.538 (PE 38:5) (Figure 2), with precursor ions separated from coeluting compounds utilizing the ramped active quadrupole.

Complex coelutions of isomers are likely to arise from samples containing VLCPUFAs, and it is these sample matrices that can prove challenging to analyze without increased separation and $\mathrm{MS}^{2}$ deconvolution technologies to purify spectra.
The $5 \mathrm{~cm} \mathrm{C18}$ column method was validated with regards to a linear range, limit of detection and quantification (LOD and LOQ), and reproducibility in terms of peak area and of retention times (Table S1 and Figure S4). It is arguable that lipidomic methodologies are rarely truly quantitative, especially if only one standard is used per class. Owing to variable ion suppression due to matrix effects and a lack of knowledge of response factors even within a lipid class, which can vary substantially due to acyl chain length and degree of unsaturation, with response deviations more pronounced when fragmentation is employed, ${ }^{15}$ relative fold changes are usually the most accurate way to report data. It is possible, however, to quantify in lipidomics if multiple lipids per class are used (a bracketing approach ${ }^{16}$ ) or, more accurately, if heavy standards for each lipid to be quantified are spiked; however, this can often be prohibitively expensive. With regards to validation, peak area \%RSD was generally maintained below $5 \%$ on triplicate injections; however, this was found to deviate significantly at the lower range of the calibration. The linear range measured was found to be in the order of $10^{3}$ for most compounds, with linearity tailing off roughly above an area of $10^{6}$, depending on peak width. Assuming linearity to the LOQ the linear range on average was found to be $10^{3.75}$. The linear region was found to have an $R^{2}$ value of above 0.99 for phospholipids; however, neutral lipids displayed reduced values, possibly owing to matrix suppressive effects due to the added sample matrix and eluting early from the short column.

This would indicate that neutral lipids are more robustly analyzed using a longer C18 column. Retention time stability was determined by six replicate injections on two separate days at two concentrations. Intraday variation was found on average to be 0.43 and $0.36 \%$ on day one and two, respectively, with the average interday variation found to be $2.37 \%$.

Development of a Neutral Lipid C18 Method. Due to the nature of salmon flesh samples, which contain high levels of triacylglycerol (TAG), it was necessary to develop a method capable of more in-depth analysis of this and other neutral lipids. Owing to the reduced resolution and selectivity on the short C18 column, we decided to utilize the longer C18 column $(15 \mathrm{~cm})$, which had demonstrated greater separation with regards to TAG. The separation conditions were found to be optimal, utilizing the same modifier solvent as the total lipid separation, methanol/acetonitrile $(80: 20, \mathrm{v} / \mathrm{v})$ with $0.15 \%(\mathrm{w} /$ v) ammonium formate and $0.1 \%(\mathrm{v} / \mathrm{v})$ formic acid; however, other combinations of methanol and acetonitrile were tested (Figure S5), as were substituting acetonitrile for isopropanol. It was found that isopropanol favored the separation of larger TAG molecules, while acetonitrile favored the separation of smaller TAGs although both solvent systems resulted in coelution of TAG species. However, depending on the oil being analyzed, the choice of mobile phase can be modified to better effect separation of the desired and most predominant TAG species. The addition of $\mathrm{MS}^{\mathrm{e}}$ and Sonar, however, allows for coelution to be mitigated to some extent. A blend of commercial Calanus finmarchicus oil and cod liver oil was used as a test mixture to determine the ability of the method to isolate individual TAGs and wax esters and obtain pure spectra from individual compounds. The method is capable of separating wax esters from TAG (Figure 3) and partially and fully separating isobaric TAG species (Figure S6). Those TAG species that do not fully separate may be more quantitatively 


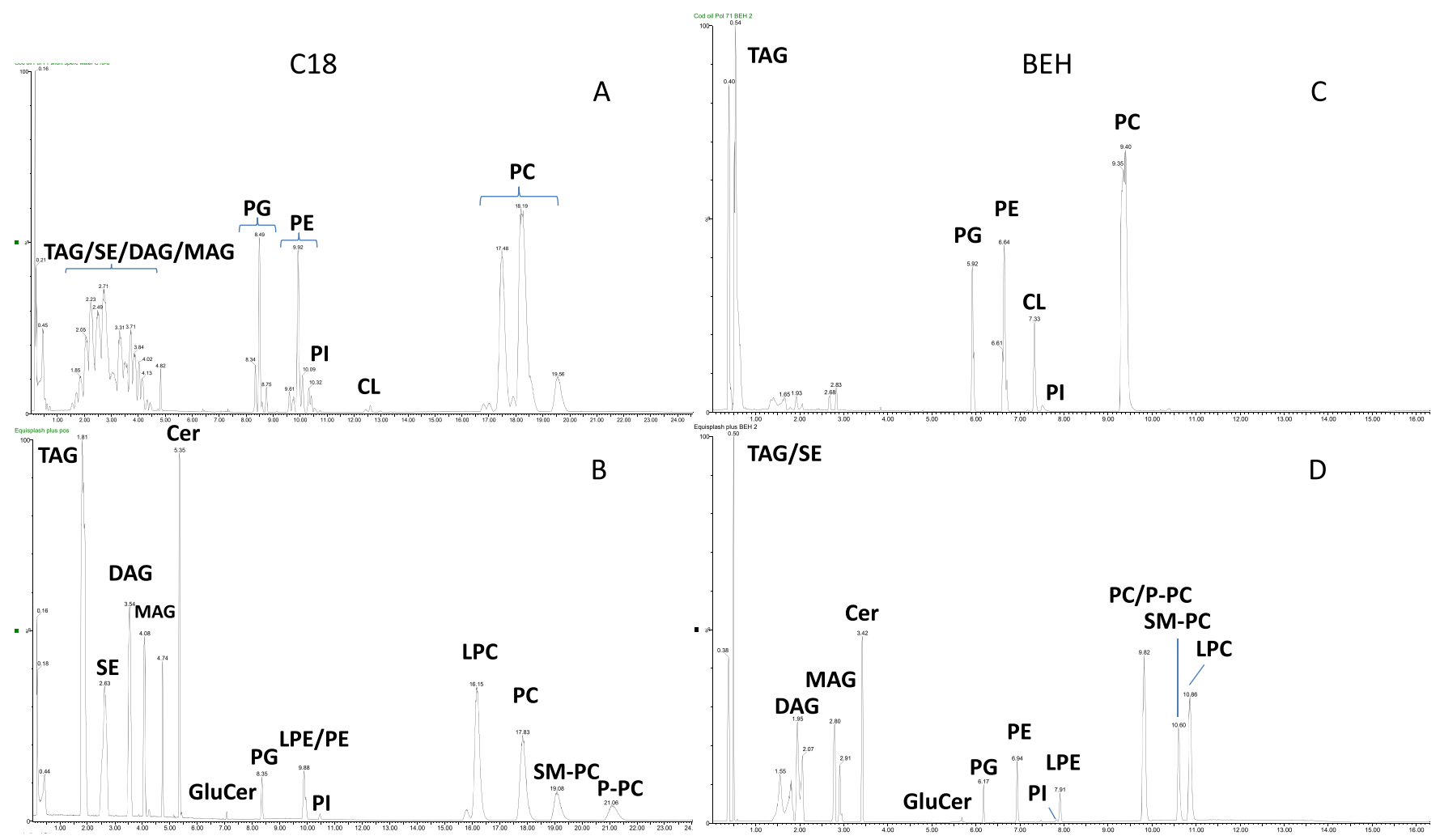

Figure 1. Comparison between the BEH and HSS $5 \mathrm{~cm} \mathrm{C18} \mathrm{columns.} \mathrm{The} \mathrm{C} 18$ column is capable of providing both interclass and intraclass separation as is demonstrated in panels (A) and (B) when a mixed standard ((A, C), Pol 71 plus cod liver oil; (B, D) Equisplash mix) is run. This contrasts with the $(\mathrm{C}, \mathrm{D}) \mathrm{BEH}$ column, whereby minimal intraclass separation is achieved. The peak shape of choline-containing lipids with the $\mathrm{C} 18$ column is somewhat broader when compared with the BEH column; however, for complex lipidomes, the intraclass selectivity improves the confidence of lipid identifications and the resolution of lipid isomers.

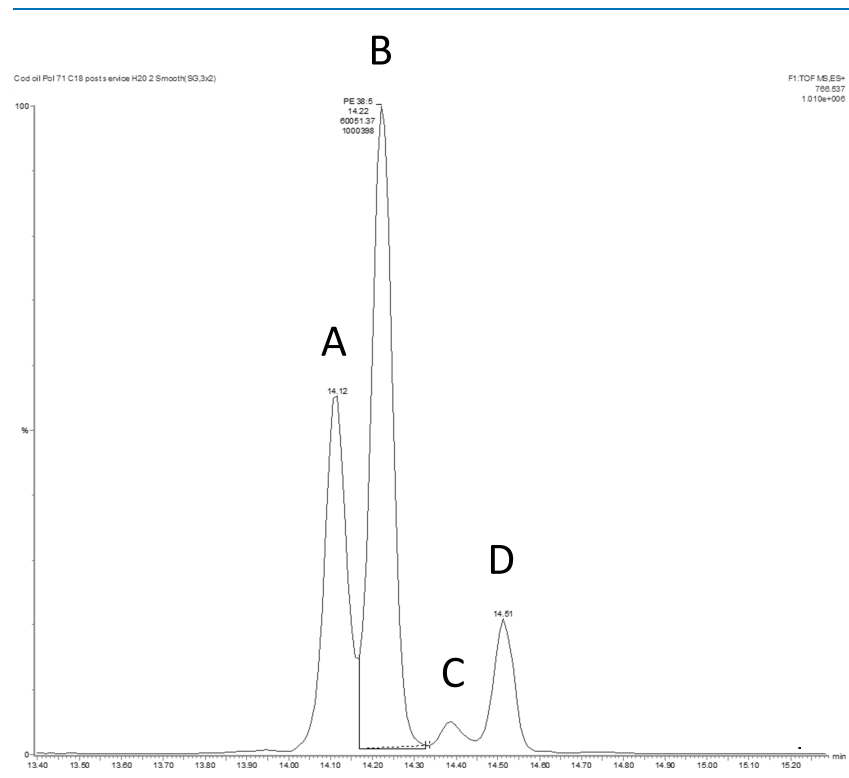

Figure 2. C18 column separation of PE 38:5 fatty acid isomers $(\mathrm{m} / \mathrm{z}$ 766.538). Peaks were identified as (A) PE 20:4/18:1 (PE 22:6/16:0 isotope overlap), (B) PE 22:5/16:0, (C) fatty acids not determined, and (D) PE 20:3/18:2.

compared by using the high energy $\left(\mathrm{MS}^{2}\right)$ function as this equates to the neutral loss of a fatty acid.

Comparison of Salmon Flesh under Different Dietary Conditions. Salmon were fed two diets: a control diet based on an industry standard feed formulation containing a mixture of rapeseed oil and fish oil and a diet formulated with the novel EPA (20:5 n3) and DHA (22:6 n3) containing Camelina sativa oil, which contains substantial quantities of $18: 3 \mathrm{n} 3$. The control diet contained approximately 49.1\% (mol \%) monounsaturates, predominantly 18:1 n9. The GM feed contained approximately double the DHA content of the control feed at 5.3\%, 5.4 times the DPA $(22: 5 \mathrm{n} 3)$ content at $3.8 \%$ and slightly elevated EPA levels at $5.7 \%$. The GM diet was also highly enriched in the fatty acid 18:3 n3 at $19.1 \%$. The salmon flesh samples contained several lipid classes (Figure 3), of which TAG was the predominant lipid. The phospholipids $\mathrm{PC}$ and PE were the most abundant polar lipids found within the tissue with cardiolipin (CL), phosphatidylinositol (PI), and sphingomyelin (SM) also detected, albeit at lower levels. The flesh of salmon fed the GM oil diet demonstrated a distinct lipid profile enriched in polyunsaturated fatty acids such as 20:5 and 22:6, which clustered this diet away from the control diet (Figures 4 and 5). The control diet was distinguished by its increased incorporation of the fatty acids 16:0, 18:0, 18:1, and 18:2, which are characteristic of native terrestrial seed oils. The TAGs are the principle drivers of class separation, partly due to being the most abundant lipids, and on average, demonstrating the greatest fold changes. The breakdown of the fatty acid composition of each lipid class, where available, is shown in Table S2. Due to the complexity of the sample matrix and large number of isomers present, multiple fatty acid combinations could be constructed from the detected neutral losses using Sonar for TAG and the deprotonated fatty acid product ions from phospholipids in negative mode. This was due to peaks containing multiple isomers, with the isomeric 


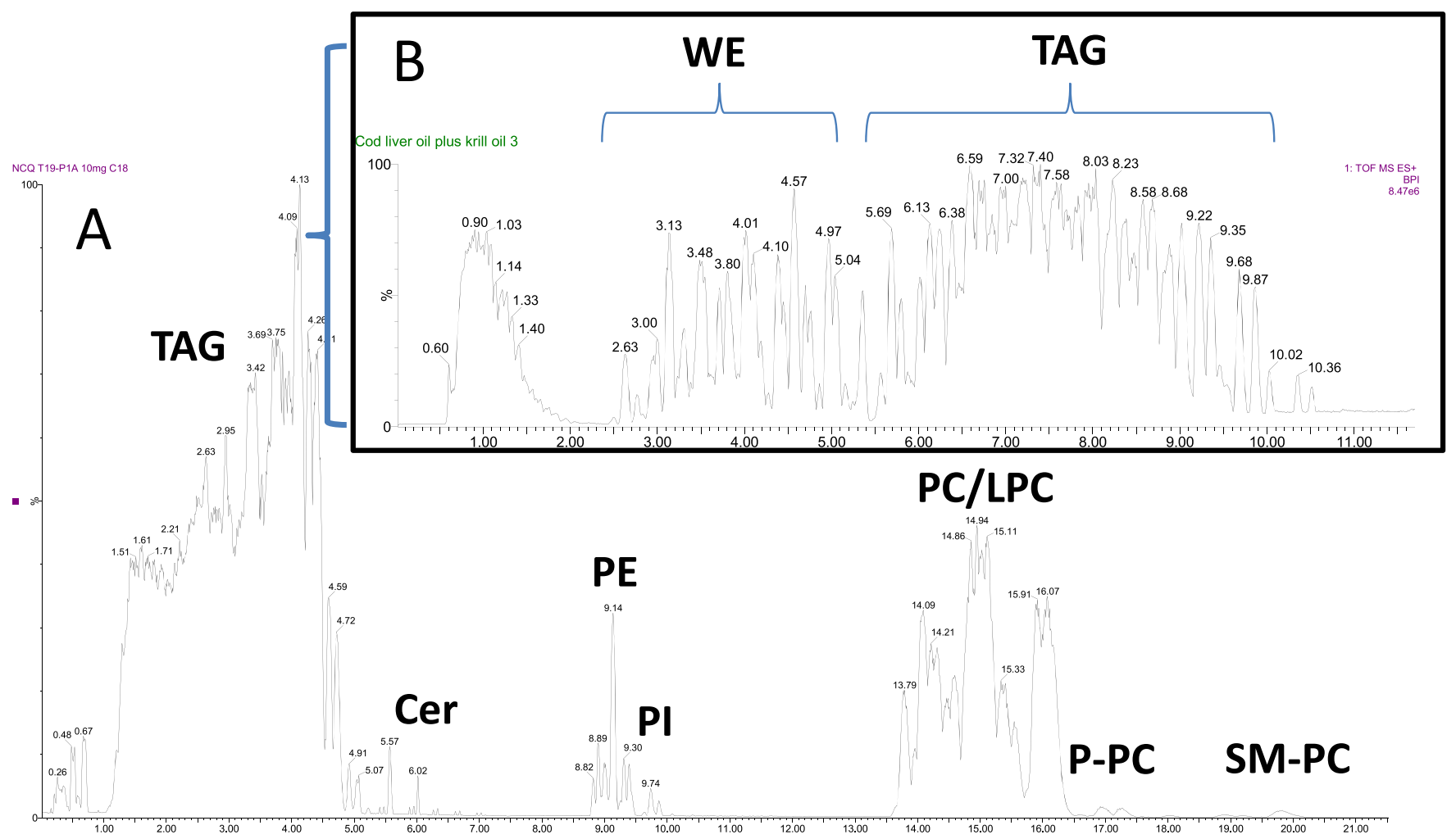

Figure 3. (A) Representative trace of a salmon flesh lipid profile in positive mode. Neutral lipids, specifically TAG, dominate the lipid profile. This can be sent to waste, with a separate diluted sample run on the $15 \mathrm{~cm}$ HSS C18 column. PC is the second most abundant class followed by PE. The intraclass separation within the PC fraction indicates the diversity present within the lipid classes, which may be missed with HILIC-type separations, especially for VLCPUFA-containing lipidomes. (B) Owing to the abundance of TAG in salmon flesh, a separate neutral lipid separation was carried out on the HSS $15 \mathrm{~cm} \mathrm{C18} \mathrm{column.} \mathrm{A} \mathrm{mixture} \mathrm{of} \mathrm{cod} \mathrm{liver} \mathrm{oil} \mathrm{and} \mathrm{krill} \mathrm{oil} \mathrm{was} \mathrm{used} \mathrm{to} \mathrm{demonstrate} \mathrm{the} \mathrm{improved} \mathrm{intraclass} \mathrm{separation}$ efficiency achieved on a longer C18 column, as is improved the peak shape and separation from other neutral classes, such as wax esters.

resolution of each compound in this complex matrix difficult to achieve.

The storage lipids appear to reflect the greater availability of either the polyunsaturated fatty acids or the monounsaturated fatty acid 18:1 n9 within the diet and appear to be enriched to a greater extent when compared to the phospholipids. The TAG fraction found within the GM diet not only appears to be enriched in the number of TAG molecules containing a VLCPUFA but also contains a greater number of polyunsaturated fatty acids affixed to the glycerol backbone. This, however, appears limited to only two acyl positions, with the third solely reserved for either saturated, monounsaturated, or polyunsaturated $\mathrm{C} 16$ or $\mathrm{C} 18$ fatty acids. It also appears that longer-chain TAG containing above 58 carbons is the most responsive to the input of VLCPUFA, potentially accumulating this large and highly unsaturated TAG to a greater extent than the original seed oil. ${ }^{10}$ However, these TAGs are not the most abundant in absolute terms with 56- and 58-carbon TAGs generally found in greater abundance, as is also found within the seed oil, displaying fold changes in the range of 1.5-2.5. Again, care should be taken when comparing absolute abundances of lipids without the requisite normalization.

The phospholipids, however, only show a maximum fold change of 3.06 for PC 36:3 in the GM diet, possibly indicating their role in lipid remodeling and potential membrane homeostatic regulation, and the subsequent shunting of polyunsaturated fatty acids from the polar lipid to the neutral lipid pool, or the potential bypass of polar lipid altogether. The control diet in some instances, however, exhibits larger fold changes with regards to the phospholipids, with PC 20:5/14:0 demonstrating a 5.04-fold increase, and with PC O-36:2 and PE P-34:1 showing similar fold changes of 5.63 and 4.06, respectively. Higher fold changes are, however, generally offset by the lower absolute abundance of the lipids. Within PC, the control diet flesh was predominantly enriched in the shorter chain, less unsaturated fatty acids, with those PC species demonstrating the highest fold change or greatest abundance containing $\mathrm{C} 16$ or $\mathrm{C} 18$ fatty acids, or generally being less unsaturated than those found within the GM oil diet. PE and PI, however, are less clear cut, with PE compounds within the control diet, which exhibit large fold changes but low abundances, containing 16:0 and 18:1, while those demonstrating larger abundances contain EPA and DHA, offering comparable levels of unsaturation within this lipid class to the GM diet. The GM oil diet, however, does result in the modification of larger PE species on average. The PE species also reflected the diet with the most abundant lipid within this species containing 18:3 for the GM oil diet and 18:1 for the control diet, both bound to DHA.

PI was also notable in that several species appear significantly modified in the control diet, whereas only one was detected within the GM diet. Just over half of the PI species in the control diet were enriched in the shorter $\mathrm{C} 16$ and $\mathrm{C} 18$ fatty acids, whereas the remaining lipids were found to contain DHA and 20:4. This was in contrast to the GM diet, which only displayed elevated levels of 18:3 containing PI, correlating with the increased abundance of this fatty acid found within the diet. The cardiolipins are likely to be more 

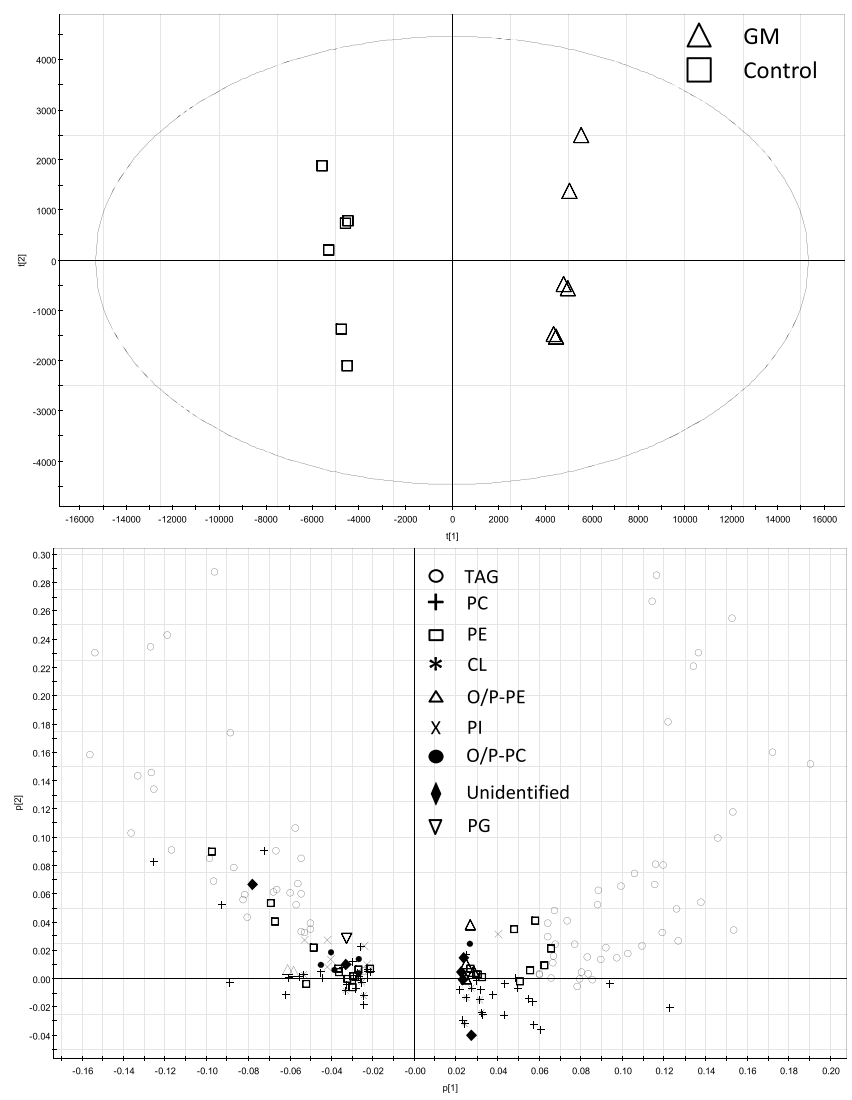

Figure 4. A multivariate PCA and score plot demonstrating the separation between Camelina sativa-supplemented salmon and a control diet. The flesh of the two treatments is predominantly separated along the first component, which explains $89.6 \%$ of the total variance. This separation is predominantly driven by their TAG profiles, while PC and PE are the main drivers of separation within the polar lipid fraction.

structurally related to the PIs and PGs owing to their shared precursor pool, with the lack of observable PI alterations within the GM diet possibly being attributable to the deposition of VLCPUFA into the larger CL molecules. The CL pools of both diets demonstrated increases in $\mathrm{CL}$ containing VLCPUFA; however, the GM diet consistently produced more unsaturated lipids within this species, likely containing 18:3, EPA, and DHA. Again, the CL species within the control diet were found to be more likely to contain shorter chain fatty acids such as 18:1.

When compared with previous works, the current findings corroborate the observation that within salmon, flesh lipid composition generally reflects the diet. ${ }^{13,17,18}$ It therefore appears beneficial to supplement diets with increased EPA and DHA to observe a concomitant increase within the flesh. In contrast, brain EPA and DHA contents appear to be tightly regulated with little variance regardless of diet. Interestingly however, in tissues known to be rich in EPA such as liver, the EPA and DHA enrichment does not match that found within the diet. Thus, liver EPA and DHA appear to be enriched regardless of the feed content, with low EPA and DHA content within the feed still yielding substantial quantities within the liver. When C18:3 n3 levels are abundant within the feed however, EPA and DHA enrichment within the liver drops drastically, indicating a role of the fatty acid in the biosynthesis of the longer chain polyunsaturates. The role of C18:3 n3 and

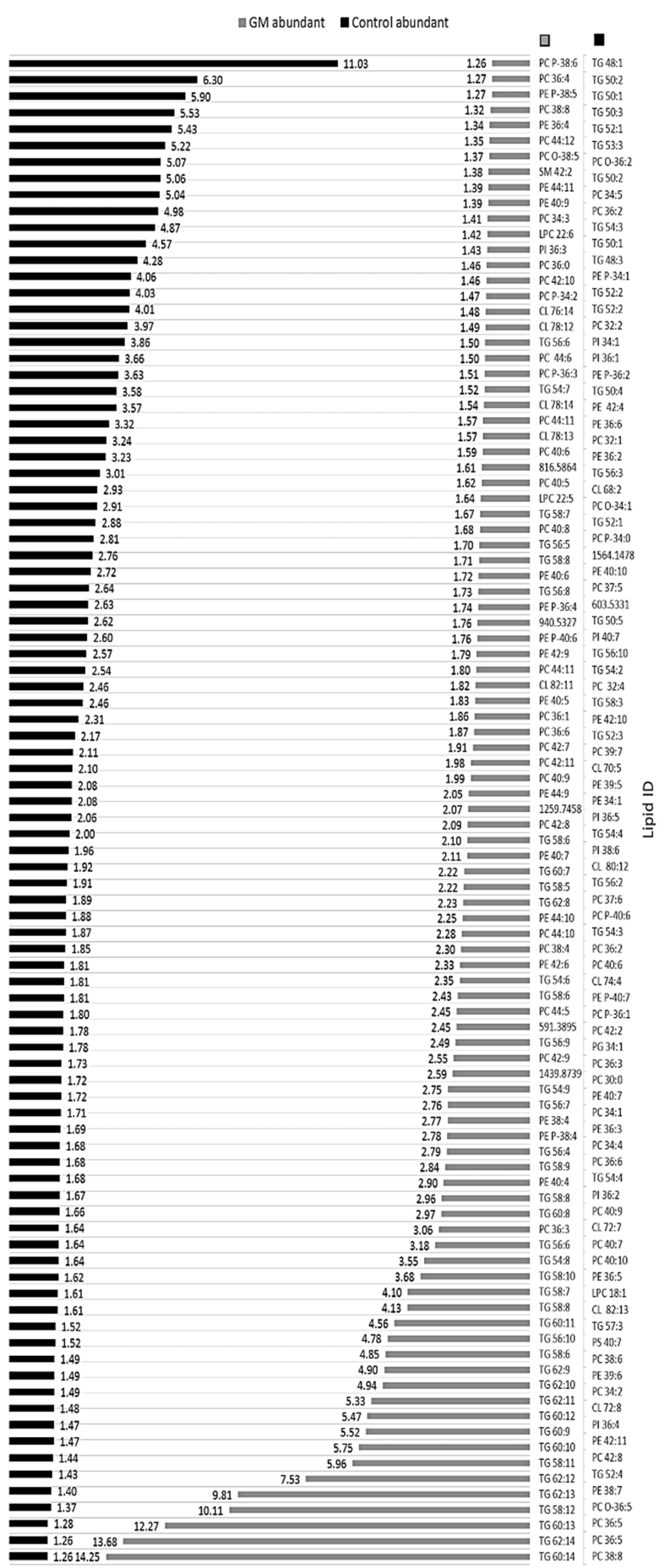

Figure 5. Lipid fold changes $(p<0.05$, fold change $>1.25)$ responsible for the separation of the two lipidomes under different dietary conditions. Those fish fed a diet supplemented with the GM oil were found to contain substantial increases in polyunsaturated TAGs such as TG 60:14 and TG 62:14. In general, the majority of lipids with an increased abundance were those containing polyunsaturated fatty acids, whereas the control diet was found to be more enriched in standard oilseed fatty acids, such as 18:1 and 18:2.

its potential effect within the liver have been indirectly corroborated, ${ }^{19}$ with an inverse gradient of fish oil to plant oil revealing that, on initial observation, diet fatty acid composition is mirrored by the liver. However, similar to the previous study, C18:3 n3 levels are increased as dietary plant oils are increased, indicating that situations whereby C18:3, 
Table 2. Summary of Methods Used within Lipidomics

\begin{tabular}{|c|c|}
\hline $\begin{array}{c}\text { sample } \\
\text { introduction }\end{array}$ & benefits/drawbacks \\
\hline \multicolumn{2}{|l|}{ UHPLC } \\
\hline $\begin{array}{l}\text { reverse } \\
\text { phase }\end{array}$ & $\begin{array}{l}\text { + provides separation based on class and provides acyl } \\
\text { selectivity } \\
+ \text { usually results in class coelution } \\
+ \text { robust and reliable } \\
\text { - may suffer from variable ionization throughout } \\
\text { chromatogram }\end{array}$ \\
\hline HILIC & $\begin{array}{l}\text { + provides separation based on class. Usually provides discrete } \\
\text { class separation } \\
+ \text { can provide more accurate quantitation through bracketing } \\
\text { and single standard approaches } \\
\text { - limited acyl selectivity }\end{array}$ \\
\hline \multicolumn{2}{|r|}{ ( } \\
\hline $\mathrm{BEH}$ & $\begin{array}{l}\text { + provides separation based on class; usually provides discrete } \\
\text { class separation. } \\
+ \text { can provide rapid class-based lipid separation. } \\
\text { - limited acyl selectivity for most current SFC column } \\
\text { chemistries. }\end{array}$ \\
\hline HSS C18 & $\begin{array}{l}\text { + provides separation based on class and provides a degree of } \\
\text { acyl selectivity. } \\
+ \text { usually provides discrete class separation. } \\
+ \text { can provide more accurate quantitation through bracketing } \\
\text { and single standard approaches. } \\
\text { - chromatographic behavior is sensitive to mobile phase and } \\
\text { SFC parameters. }\end{array}$ \\
\hline $\begin{array}{l}\text { direct } \\
\text { infusion }\end{array}$ & $\begin{array}{l}\text { + can provide rapid "shotgun" lipidomics analysis. } \\
\text { traditionally used with targeted triple quadrupole methods. } \\
\text { + can be quantitative with bracketing. } \\
\text { - limited structural detail. }\end{array}$ \\
\hline $\begin{array}{c}\text { MALDI/ } \\
\text { DESI }\end{array}$ & $\begin{array}{l}\text { + can provide in situ lipidomics imaging of samples. } \\
\text { traditionally coupled to a TOF detector. } \\
\text { - usually not quantitative. } \\
\text { - usually limited structural information. }\end{array}$ \\
\hline
\end{tabular}

EPA, and DHA are depleted in feed can result in VLCPUFA accumulation within certain tissues, such as the liver. However, the contrary has also been shown ${ }^{13}$ with DHA liver concentrations seemingly static regardless of feed DHA and C18:3 n 3 concentrations.

The studies, however, only ascertained the total fatty acid composition, and whether TAGs and PCs are the primary lipids, which are altered, is yet to be determined, as is the role of $\mathrm{C} 18: 3 \mathrm{n} 3$ and its apparent regulatory role. Through the development of these methods, we aim to further our insight into lipid metabolism within finfish species and address which diets are most effective at promoting VLCPUFA production within these commercial species. It is evident that lipid remodeling within different tissues in response to diet is likely more complicated than traditional methodologies have revealed.

The methods outlined here compare favorably with those currently used within the field of lipidomics, as illustrated in Table 2. In comparison to the standard methods, i.e., either reverse phase or HILIC, the present method provides a blend of both by offering separation similar to HILIC with the acyl selectivity found within reverse phase. It is to be noted, however, that reverse phase chromatography in general is more forgiving in terms of robustness, with the SFC mobile phase and system parameters requiring attention to detail to prevent retention time drift. Regarding quantitation, the HSS C18 method potentially allows for more accurate quantification owing to discrete class separation, minimizing interclass ion

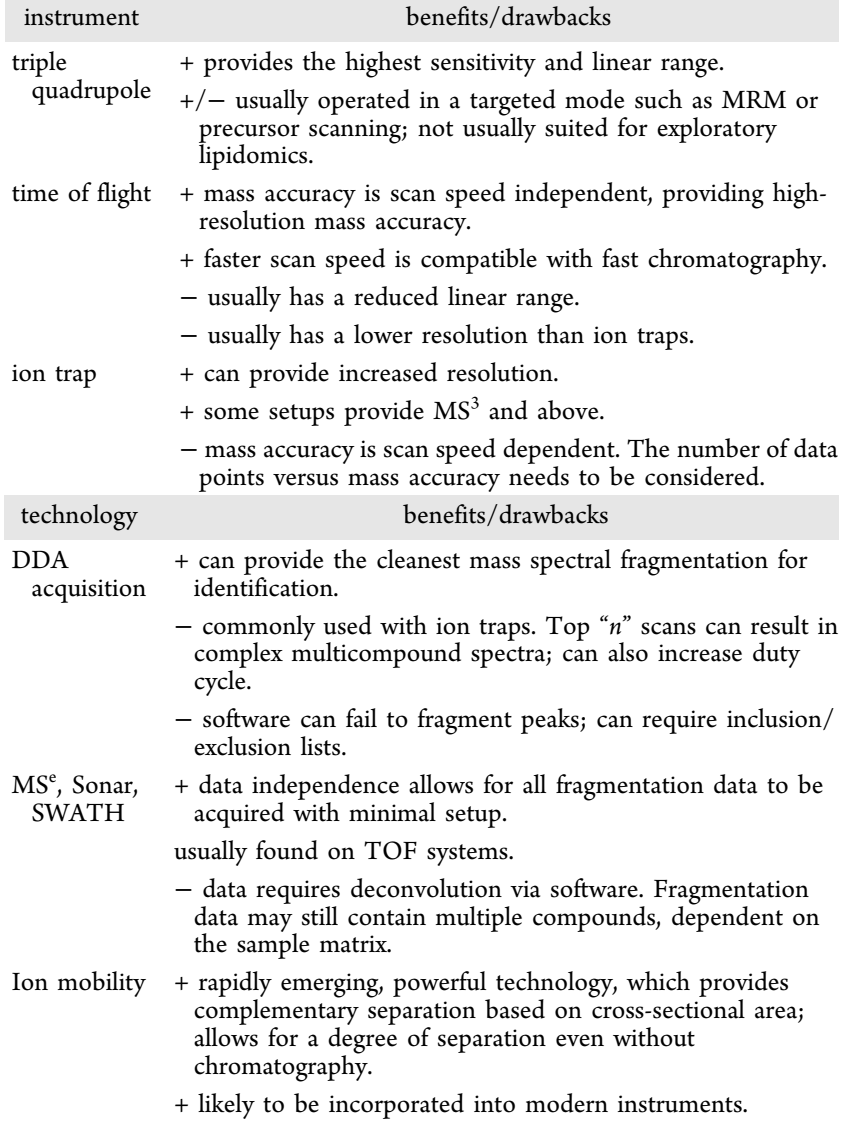

suppression effects, as may be found within reverse phase systems. The addition of $\mathrm{MS}^{\mathrm{e}}$ and Sonar allows for data independent acquisition, allowing for simplified method development and the collection of large amounts of data, which can be interrogated postacquisition. However, with the reduced linear range of TOF machines, depending on the sample matrix, several methods and columns must be run to acquire data within the linear range for different classes, and in different polarities, to allow for suitable fragment acquisition. Currently, while a system capable of providing both quantitative data, broad coverage of classes, and depth of analysis is unlikely, with the development in column chemistry, and the implementation of ion mobility, more comprehensive data sets will likely be possible as more complex duty cycles become compatible with fast chromatography.

\section{CONCLUSIONS}

In summary, the SFC method developed expands on the current strengths of supercritical fluid chromatography, namely, the normal phase like class separation and reduction in solvent usage. It also allows for the ability to either rapidly screen with BEH-based columns, leading to discrete classbased separation, or improve intraclass separation through the usage of an HSS C18 column while still retaining discrete class separation, which aids in compound identification. When coupled to either $\mathrm{MS}^{\mathrm{e}}$ or an active quadrupole, compound identities can be obtained in a data-independent fashion, 
potentially resulting in less complex method development owing to the lack of inclusion/exclusion lists and the missing of less abundant peaks. The use of two methods ensured the optimal separation of both neutral lipids and phospholipids; however, a generic method could be utilized depending on the matrix complexity and abundances of the different classes. The C18 phase also provided some ability to resolve isomers, allowing for more accurate assessments of both fold changes and identities within the studied lipid matrix, which was enriched in polyunsaturated fatty acids. Such methods are, however, used in an exploratory fashion, providing a list of candidate compounds for further investigation by more targeted methodologies, which would determine whether compounds of interest display isomeric differences in both fatty acid composition and sn position. It was found that, in general, flesh lipid composition reflected the fatty acids found within the diet, with fish fed on the GM oil diet being found to produce lipids more enriched in VLCPUFAs such as EPA and DHA and the control diet enriched in the fatty acids associated with terrestrial oil seeds such as $18: 1$.

\section{EXPERIMENT SECTION}

Materials. Methanol and acetonitrile were sourced from Rathburn Chemicals (Walkerburn, UK), isopropanol and formic acid were sourced from Biosolve (Valkenswaard, Netherlands), and water and ammonium formate were purchased from Merck (Dorset, UK), with all solvents being LC-MS grade. $\mathrm{CO}_{2}$ (99.8\% industrial grade) was purchased from BOC (Grangemouth, UK). The Equisplash mix, glucosyl C12 ceramide, and 18:1/18:1 plasmalogen were obtained from Avanti Polar lipids (Alabama, USA), and Pol mix 71 was obtained from Laroden (Solna, Sweden). Triacylglycerol and wax ester blends were sourced from commercial cod liver oil and Calanus finmarchicus oil.

Atlantic Salmon Growth Conditions. All procedures were conducted in compliance with the Animals Scientific Procedures Act 1986 (Home Office Code of Practice) and in accordance with the regulations set forward by the Directive 2010/63/EU of the European Parliament and of the Council of 22 September 2010 on the protection of animals used for scientific purposes. Additionally, the experimental protocol was approved by the Animal Welfare and Ethical Review Board at the University of Stirling [AWERB(16-17)83]. A total of 900 post-smolt Atlantic salmon (Salmo salar L.) with an average initial body weight of $187.2 \pm 1.9 \mathrm{~g}$ (mean \pm SD) were distributed into six $5 \mathrm{~m}^{3}$ seawater floating pens (150 fish per pen) and fed one of two experimental diets in triplicate from June 2018 to November 2018 with average fish final weight of $1941.8 \pm 156.3$ (mean \pm SD) with no significant difference between dietary treatments. The diets were isolipidic (36\%) and isoproteic (30\%) and formulated to contain either a blend of vegetable (rapeseed oil) and fish oil (2:1, by vol.), the control diet, or a de novo oil, rich in both EPA and DHA, obtained from GM Camelina sativa (Table 3), the GM diet. At the end of the experimental period, six fish per pen were humanely euthanized by anesthetic overdose of metacaine sulfonate $(>150 \mathrm{mg} / \mathrm{L})$, and the flesh portion corresponding to the Norwegian quality cut (NQC) was collected and immediately frozen at $-70{ }^{\circ} \mathrm{C}$ prior to lipid extraction.

Salmon Flesh Lipid Extraction. Total lipid of flesh was extracted from the NQC $(n=6)$ according to Folch et al. ${ }^{20}$ Briefly, approximately $1 \mathrm{~g}$ of homogenized NQC was extracted with $20 \mathrm{~mL}$ of chloroform/ methanol $(2: 1, \mathrm{v} / \mathrm{v})$ by blending
Table 3. Control and GM Oil Diet Compositions

\begin{tabular}{|c|c|c|}
\hline contents & control & GM \\
\hline \multicolumn{3}{|l|}{ feed ingredients (\%) } \\
\hline fish meal, BioMar A/S & 7.5 & 7.5 \\
\hline soy protein concentrate & 10.1 & 10.1 \\
\hline maize gluten & 5.0 & 5.0 \\
\hline pea protein & 5.4 & 5.4 \\
\hline guar meal & 15.0 & 15.0 \\
\hline land animal protein & 10.0 & 10.0 \\
\hline wheat & 10.9 & 10.9 \\
\hline fish oil & 10.8 & \\
\hline rapeseed oil & 20.8 & \\
\hline Camelina oil (transgenic) & & 31.6 \\
\hline premix & 3.6 & 3.6 \\
\hline yttrium oxide & 0.005 & 0.005 \\
\hline \multicolumn{3}{|l|}{ fatty acid profile (\%) } \\
\hline total saturated ${ }^{1}$ & 18.1 & 14.7 \\
\hline total monoenes ${ }^{2}$ & 49.1 & 22.2 \\
\hline $18: 2 n-6$ & 15.2 & 19.6 \\
\hline $18: 3 n-6$ & 0.1 & 1.5 \\
\hline $20: 4 n-6$ & 0.4 & 1.7 \\
\hline total n-6 PUFA & 16.0 & 25.2 \\
\hline $18: 3 n-3$ & 5.8 & 19.1 \\
\hline $20: 5 n-3$ & 4.8 & 5.7 \\
\hline $22: 5 n-3$ & 0.7 & 3.8 \\
\hline $22: 6 n-3$ & 2.4 & 5.3 \\
\hline total n-3 PUFA & 15.1 & 37.7 \\
\hline
\end{tabular}

with a tissue disrupter (Ultra-Turrax; Fisher Scientific, Loughborough, UK) for $20 \mathrm{~s}$ before being left on ice for 1 h. The solution was then partitioned by the addition of $5 \mathrm{~mL}$ of $\mathrm{KCl}$ solution $(1 \%, \mathrm{w} / \mathrm{v})$, vortex mixed, then followed by centrifugation to clarify the biphasic system. The upper aqueous layer was removed and discarded with the lower chloroform layer filtered through a Whatman no. 1 filter paper and solvent evaporated under a stream of oxygen-free nitrogen before vacuum desiccation overnight. Total lipid was quantified gravimetrically and resuspended to form a stock solution of $10 \mathrm{mg} / \mathrm{mL}$ in chloroform/methanol $(2: 1, \mathrm{v} / \mathrm{v})$ containing $0.01 \%(\mathrm{w} / \mathrm{v})$ butylated hydroxytoluene as antioxidant. A 1:80 dilution was used for neutral lipid, a 1:2 dilution for positive mode phospholipid, and a 2.5 times concentration for negative mode phospholipid analyses. Samples were injected in the chloroform/methanol solution.

UPC $^{2}$-ESI-MS/MS. SFC separation was carried out on an Acquity $\mathrm{UPC}^{2}$ system (Waters, USA) with $\mathrm{CO}_{2}$ as solvent A and the cosolvent (solvent B) being either methanol/ acetonitrile $(80: 20, \mathrm{v} / \mathrm{v})$ or methanol/acetonitrile/water $(75: 20: 5, \mathrm{v} / \mathrm{v} / \mathrm{v})$ for both the $\mathrm{BEH}$ and $15 \mathrm{~cm} \mathrm{C18} \mathrm{HSS}$ column, or the $5 \mathrm{~cm} \mathrm{C} 18$ column, respectively. Both solvent systems contained ammonium formate $(0.15 \% \mathrm{w} / \mathrm{v})$ and $0.1 \%$ (v/v) formic acid. The make-up solvent consisted of methanol/isopropanol (80:20, v/v) containing 0.1\% ammonium formate $(\mathrm{w} / \mathrm{v})$ and was tee'd in after the column. The strong wash solution consisted of methanol/acetonitrile $(80: 20, \mathrm{v} / \mathrm{v})$, while the weak wash and seal wash comprised of methanol. Three columns were used in this study: a Viridis (Waters, USA) $\mathrm{BEH} 100 \times 3.0 \mathrm{~mm}, 1.7 \mu \mathrm{m}$ particle size column; a Viridis C18 HSS $150 \times 2.1 \mathrm{~mm}, 1.8 \mu \mathrm{m}$ particle size column; and a Viridis C18 HSS $50 \times 2.1 \mathrm{~mm}, 1.8 \mu \mathrm{m}$ particle column. The gradient runs were optimized for each application and are shown in Table 4. 
Table 4. SFC Gradient Conditions for the Three Columns Chosen for this Study ${ }^{a}$

\begin{tabular}{|c|c|c|c|c|c|c|c|}
\hline \multicolumn{2}{|c|}{ BEH $100 \times 2.1$} & \multicolumn{4}{|c|}{ C18 HSS $150 \times 3.0$} & \multicolumn{2}{|c|}{$\begin{array}{c}\text { C18 HSS } 50 \times \\
3.0\end{array}$} \\
\hline time & B \% & time (TAG) & B \% & time (PL) & B \% & time & B \% \\
\hline 0 & 2 & 0 & 2 & 0 & 2 & 0 & 2 \\
\hline 0.75 & 2 & 1.5 & 2 & 5.0 & 2 & 2.5 & 2 \\
\hline 10.75 & 50 & 16.0 & 20 & 20.0 & 50 & 18.70 & 64 \\
\hline 13.0 & 50 & 17.0 & 20 & 26.0 & 50 & 24.00 & 64 \\
\hline 16.0 & 2 & 20.0 & 2 & 32.0 & 2 & 27.50 & 2 \\
\hline 18.0 & 2 & 22.0 & 2 & 35.0 & 2 & 29.50 & 2 \\
\hline
\end{tabular}

${ }^{a}$ The $15 \mathrm{~cm} \mathrm{C18}$ column was used for both the neutral lipid (TAG) and phospholipid (PL) methods.

The back pressure regulator was set at 1500 psi with the flow rate being set at $1.5,0.7$, and $1.2 \mathrm{~mL} / \mathrm{min}$ for the $\mathrm{BEH}, \mathrm{C} 18$ $(15 \mathrm{~cm})$, and $\mathrm{C} 18(5 \mathrm{~cm})$ columns, respectively. The column temperature was held at $50{ }^{\circ} \mathrm{C}$ for both the $\mathrm{BEH}$ and $\mathrm{C} 18$ (15 $\mathrm{cm})$ columns, while it was held at $52.5{ }^{\circ} \mathrm{C}$ for the $\mathrm{C} 18(5 \mathrm{~cm})$ column. Then, $1.5 \mu \mathrm{L}$ was injected onto the system per sample.

The UPC ${ }^{2}$ system was attached to a Xevo G2-XS Q-TOF (Waters, USA) mass spectrometer. The system was operated with an ESI source in both positive and negative modes with the source settings for both positive and negative mode being 3 $\mathrm{kV}$ capillary voltage, $28 \mathrm{~V}$ sampling cone voltage, a source offset of 80 , a source and desolvation temperature of 120 and $300{ }^{\circ} \mathrm{C}$, respectively, and a cone and desolvation gas flow of 50 and $1000 \mathrm{~L} / \mathrm{h}$, respectively. The mass spectrometer was operated in the data-independent $\mathrm{MS}^{\mathrm{e}}$ mode, which alternates between a high energy and low energy scan. The scan range was set between 200 and $1600 \mathrm{Da}$ at $0.25 \mathrm{sec} / \mathrm{scan}$ with a low energy collision cell voltage of $2 \mathrm{~V}$ and a ramped voltage of 28-40 V for the high energy scan. For Sonar, the scan range was reduced: for positive mode, it was set at 300-1200 Da with a scan speed of $0.3 \mathrm{scan} / \mathrm{sec}$ with the quadrupole start mass set at $300 \mathrm{Da}$ and end mass at $1200 \mathrm{Da}$ with a mass width of $15 \mathrm{Da}$. For negative mode, the scan range was set to 200$1060 \mathrm{Da}$ with a scan time of $0.3 \mathrm{scan} / \mathrm{sec}$ with a quadrupole start mass of $560 \mathrm{Da}$, end mass of $1060 \mathrm{Da}$, and a mass window of $15 \mathrm{Da}$. The lock mass compound was leu-enkephalin, and this was infused at a rate of $10 \mu \mathrm{L} / \mathrm{min}$ throughout the run to correct for mass deviations.

Method Validation. The method was validated using the Equisplash standard mix, which contains 13 standards at 100 $\mu \mathrm{g} / \mathrm{mL}$. Added to this were $18: 1 / 18: 1$ plasmalogen, as was glucosyl C12 ceramide, which was kept at a constant concentration, and was used as a reference standard. Eight calibration levels were used $(100 \mu \mathrm{g} / \mathrm{mL}, 1: 2,1: 10,1: 100$, $1: 200,1: 500,1: 1000$, and $1: 1500$ dilutions) with a constant amount of a $10 \mathrm{mg} / \mathrm{mL}$ salmon flesh lipid extract added as a sample matrix to each standard.

Data Processing. The UPC ${ }^{2}$ and mass spectrometer were operated using Masslynx V4.2 (Waters, USA). Calibration data was analyzed using Masslynx, while salmon data acquired with $\mathrm{MS}^{\mathrm{e}}$ and Sonar were analyzed with Progenesis QI v 3.0 (Nonlinear Dynamics, UK) and $\mathrm{MS}^{\mathrm{e}}$ viewer (Waters, USA). Simca-P v12.0 (Umetrics, Sweden) was used for multivariate analysis with OPLS-DA used to discriminate between classes and with parametric scaling used to partially compensate for the wide range of compound abundances. The subsequent significant mass spectral features were identified using an S- plot with features of interest reimported back into Progenesis for compound identification.

\section{ASSOCIATED CONTENT}

\section{Supporting Information}

The Supporting Information is available free of charge at https://pubs.acs.org/doi/10.1021/acsomega.0c02631.

Supporting information includes the comparison of a $\mathrm{BEH} \mathrm{C} 18$ column (Figure S1), the influence of silyl ether formation on retention time (Figure S2), a comparison of the 15 and $5 \mathrm{~cm} \mathrm{C18}$ columns (Figure S3), the calibration curves (Figure S4) and validation data for the method (Table S1), a comparison of modifier solvent ratios on TAG selectivity (Figure S5), illustration of partial and full TAG isomer separation (Figure S6), and fatty acid composition data of identified compounds (Table S2) (PDF)

\section{AUTHOR INFORMATION}

\section{Corresponding Author}

Richard Broughton - Institute of Aquaculture, Faculty of Natural Sciences, University of Stirling, Stirling FK9 4LA, U.K.; (1) orcid.org/0000-0001-7339-2760;

Email: richard.broughton@stir.ac.uk

\section{Authors}

Douglas R. Tocher - Institute of Aquaculture, Faculty of Natural Sciences, University of Stirling, Stirling FK9 4LA, U.K.

Mónica B. Betancor - Institute of Aquaculture, Faculty of Natural Sciences, University of Stirling, Stirling FK9 4LA, U.K.

Complete contact information is available at:

https://pubs.acs.org/10.1021/acsomega.0c02631

\section{Author Contributions}

The manuscript was written through contributions from all authors, with all authors having given approval to the final version of the manuscript.

\section{Notes}

The authors declare no competing financial interest.

\section{ACKNOWLEDGMENTS}

The authors were granted early access by Waters to a beta build of Progenesis QI v3.0 for the analysis of SFC-MS/MS data. The work was supported by research grants from the UK Biotechnology and Biological Sciences Research Council (BBSRC), specifically Super Follow-On-Funding BB/ N022157/1, "Validating the commercial potential of genetically modified plant oils in aquaculture via full life-cycle replacement of marine feed ingredients", and Industrial Partnership Award BB/S005919/1 "Novel omega-3 sources in feeds and impacts on salmon health".

\section{ABBREVIATIONS USED}

VLCPUFA, very long chain polyunsaturated fatty acid; PC, phosphatidylcholine; LPC, lysophosphatidylcholine; SM-PC, sphingomyelin phosphatidylcholine; O/P-PC, plasmanyl/ plasmenyl-phosphatidylcholine; $\mathrm{PE}$, phosphatidylethanolamine; LPE, lysophosphatidylethanolamine; O/P-PE, plasmanyl/plasmenyl-phosphatidylethanolamine; PI, phosphatidylinositol; PG, phosphatidylglycerol; CL, cardiolipin; Cer, ceramide; GluCer, cerebroside; MAG, monoacylglycerol; DAG, diacylglycerol; TAG, triacylglycerol; WE, wax ester 


\section{REFERENCES}

(1) Lisa, M.; Holčapek, M. High-Throughput and Comprehensive Lipidomic Analysis Using Ultrahigh-Performance Supercritical Fluid Chromatography-Mass Spectrometry. Anal. Chem 2015, 87, 71877195.

(2) Salazar, C.; Jones, M. D.; Sturtevant, D.; Horn, P. J.; Crossley, J.; Zamann, K.; Chapman, K. D.; Wrona, M.; Isaac, G.; Smith, N. W.; Shulaev, V. Development and Application of Sub-2- $\mu \mathrm{m}$ Particle CO2 Based Chromatography Coupled to Mass Spectrometry for Comprehensive Analysis of Lipids in Cottonseed Extracts. Rapid Commun Mass Spectrom. 2017, 31, 591-605.

(3) Bamba, T.; Shimonishi, N.; Matsubara, A.; Hirata, K.; Nakazawa, Y.; Kobayashi, A.; Fukusaki, E. High Throughput and Exhaustive Analysis of Diverse Lipids by Using Supercritical Fluid Chromatography-Mass Spectrometry for Metabolomics. J. Biosci. Bioeng. 2008, 105, 460-469.

(4) Takeda, H.; Izumi, Y.; Takahashi, M.; Paxton, T.; Tamura, S.; Koike, T.; Yu, Y.; Kato, N.; Nagase, K.; Shiomi, M.; Bamba, T. Widely-Targeted Quantitative Lipidomics Methodology by Supercritical Fluid Chromatography Coupled with Fast-Scanning Triple Quadrupole Mass Spectrometry. J. Lipid Res. 2018, 59, 1283-1293.

(5) Yamada, T.; Uchikata, T.; Sakamoto, S.; Yokoi, Y.; Nishiumi, S.; Yoshida, M.; Fukusaki, E.; Bamba, T. Supercritical Fluid Chromatography/Orbitrap Mass Spectrometry Based Lipidomics Platform Coupled with Automated Lipid Identification Software for Accurate Lipid Profiling. J. Chromatogr. A 2013, 1301, 237-242.

(6) Jones, J. W.; Carter, C. L.; Li, F.; Yu, J.; Pierzchalski, K.; Jackson, I. L.; Vujaskovic, Z.; Kane, M. A. Ultraperformance Convergence Chromatography-High Resolution Tandem Mass Spectrometry for Lipid Biomarker Profiling and Identification. Biomed. Chromatogr. 2017, 31, 1-13.

(7) Laboureur, L.; Ollero, M.; Touboul, D. Lipidomics by Supercritical Fluid Chromatography. Int. J. Mol. Sci. 2015, 16, $13868-13884$.

(8) van Erp, H.; Bryant, F. M.; Martin-Moreno, J.; Michaelson, L. V.; Bhutada, G.; Eastmond, P. J. Engineering the Stereoisomeric Structure of Seed Oil to Mimic Human Milk Fat. Proc. Natl. Acad. Sci. U.S.A. 2019, 116, 20947-20952.

(9) Innis, S. M. Dietary Triacylglycerol Structure and its Role in Infant Nutrition. Adv. Nutr. 2011, 2, 275-283.

(10) Usher, S.; Han, L.; Haslam, R.; Michaelson, L. V.; Sturtevant, D.; Aziz, M.; Chapman, K. D.; Sayanova, O.; Napier, J. A. Tailoring Seed Oil Composition in the Real World: Optimising Omega-3 Long Chain Polyunsaturated Fatty Acid Accumulation in Transgenic Camelina Sativa. Sci. Rep. 2017, 7, 1-12.

(11) Petrie, J. R.; Shrestha, P.; Zhou, X.; Mansour, M. P.; Liu, Q.; Belide, S.; Nichols, P. D.; Singh, S. P. Metabolic Engineering Plant Seeds with Fish Oil-Like Levels of DHA. PLoS One 2012, 7, 1-7.

(12) Hamilton, M. L.; Powers, S.; Napier, J. A.; Sayanova, O. Heterotrophic Production of Omega-3 Long-Chain Polyunsaturated Fatty Acids by Trophically Converted Marine Diatom Phaeodactylum tricornutum. Mar. Drugs 2016, 14, 1-10.

(13) Betancor, M. B.; Li, K.; Bucerzan, V. S.; Sprague, M.; Sayanova, O.; Usher, S.; Han, L.; Norambuena, F.; Torrissen, O.; Napier, J. A.; Tocher, D. R.; Olsen, R. E. Oil from Transgenic Camelina sativa Containing over $25 \% n-3$ Long-Chain PUFA as the Major Lipid Source in Feed for Atlantic Salmon (Salmo salar). J. Geophys. Res. Oceans 2018, 119, 1378-1392.

(14) Fairchild, J. N.; Brousmiche, D. W.; Hill, J. F.; Morris, M. F.; Boissel, C. A.; Wyndham, K. D. Chromatographic Evidence of Silyl Ether formation (SEF) in Supercritical Fluid Chromatography. Anal. Chem. 2015, 87, 1735-1742.

(15) Broughton, R.; Ruíz-Lopez, N.; Hassall, K. L.; Martínez-Force, E.; Garcés, R.; Salas, J. J.; Beaudoin, F. New Insights in the Composition of Wax and Sterol Esters in Common and Mutant Sunflower Oils Revealed by ESI-MS/MS. Food Chem. 2018, 269, 7079.

(16) Brügger, B.; Erben, G.; Sandhoff, R.; Wieland, F. T.; Lehmann, W. D. Quantitative Analysis of Biological Membrane Lipids at the
Low Picomole Level by Nano-Electrospray Ionization Tandem Mass Spectrometry. Proc. Natl. Acad. Sci. 1997, 94, 2339-2344.

(17) Betancor, M. B.; Li, K.; Sprague, M.; Bardal, T.; Sayanova, O.; Usher, S.; Han, L.; Màsøval, K.; Torrissen, O.; Napier, J. A.; Tocher, D. R.; Olsen, R. E. An Oil Containing EPA and DHA from Transgenic Camelina sativa to Replace Marine Fish Oil in Feeds for Atlantic Salmon (Salmo salar L.): Effects on Intestinal Transcriptome, Histology. Tissue Fatty Acid Profiles and Plasma Biochemistry. PLoS One 2017, 12, 1-29.

(18) Foroutani, M. B.; Parrish, C. C.; Wells, J.; Taylor, R. G.; Rise, M. L.; Shahidi, F. Minimizing Marine Ingredients in Diets of Farmed Atlantic Salmon (Salmo salar): Effects on Growth Performance and Muscle Lipid and Fatty Acid Composition. PLoS One 2018, 13, 1-14.

(19) Houston, S. J. S.; Karalazos, V.; Tinsley, J.; Betancor, M. B.; Martin, S. A. M.; Tocher, D. R.; Monroig, O. The Compositional and Metabolic Responses of Gilthead Seabream (Sparus aurata) to a dietary fish oil and associated n-3 long-chain PUFA content. $J$. Geophys. Res. Oceans 2017, 118, 1010-1022.

(20) Folch, J.; Lees, M.; Stanley, G. H. S. A Simple Method for the Isolation and Purification of Total Lipides from Animal Tissues. J. Biol. Chem. 1957, 226, 497-509. 